\title{
Optimizing the Parameters of Laser Controlled Thermocracking When Cutting Glass by a Non-Rectilinear Contour
}

\author{
Vladimir Stepanovich Kondratenko ${ }^{1}$, Vladimir Yeugenyevich Borisovskiy², \\ Alexander Sergeevich Naumov ${ }^{2}$, Aleksey Konstantinovich Zobov ${ }^{2}$ \\ ${ }^{1}$ Institute of High Technologies MGUPI, Department 'Innovative Technologies in Engineering, Micro- and \\ Opto-Electronics' (MGUPI), Moscow, Russia \\ ${ }^{2}$ Moscow State University of Instrument Engineering and Computer Science (MGUPI), Moscow, Russia \\ Email: vsk1950@mail.ru, borisovskiyi ve@mgupi.ru, 8097@rambler.ru, dj.lexa.777@gmail.com
}

Received 25 March 2015; accepted 23 October 2015; published 26 October 2015

Copyright (C) 2015 by authors and Scientific Research Publishing Inc.

This work is licensed under the Creative Commons Attribution International License (CC BY). http://creativecommons.org/licenses/by/4.0/

(c) (i) Open Access

\begin{abstract}
The work is devoted to the choice of optimal parameters of laser controlled thermocracking (LCT) when cutting products with a complex curvilinear contour. With this purpose the LCT mathematical model is considered when a laser beam and coolant transfer from a rectilinear site to a nonrectilinear contour with given radius $R$. The obtained findings allow proper adjustment of LCT modes to ensure a higher quality of cutting the glass.
\end{abstract}

\section{Keywords}

Laser Controlled Thermocracking (LCT), $\mathrm{CO}_{2}$-Laser

\section{Introduction}

Recently the LCT method has gained a wide application in various branches of production due to a number of indisputable advantages, among which in the first place it should highlight:

- high speed of cutting-up to $1000 \mathrm{~mm} / \mathrm{sec}$,

- high accuracy-up to 10 microns,

- high endurance of the product (5.5 times as high as the strength after mechanical processing of the edge [1]),

- high quality which does not require additional processing,

- wastelessness of the cutting process.

We remind that the LCT method essentially means the formation of a separating partial crack in the surface

How to cite this paper: Kondratenko, V.S., Borisovskiy, V.Y., Naumov, A.S. and Zobov, A.K. (2015) Optimizing the Parameters of Laser Controlled Thermocracking When Cutting Glass by a Non-Rectilinear Contour. Optics and Photonics Journal, 5, 295-302. http://dx.doi.org/10.4236/opj.2015.510027 
layer which then propagates in the material according to a preset separation contour after laser radiation and the coolant has been applied. Physical model of the LCT is shown in Figure 1.

The separating cracking mechanism during LCT of the fragile nontransparent plates for the given length of the material laser radiation is the following.

When irradiating the surface of brittle material with laser radiation of IR range, there is considerable stress compression in the external layers, which, however, does not lead to its destruction. When applying the coolant after the laser beam on the boil front there is a sharp local cooling of the surface of the material at the cutting line. The created temperature gradient determines the arising tensile stresses in the material surface layers, which provides the propagation of a crack of a given depth. The crack penetration depth is conditioned by the distribution of thermoelastic stresses depending on a number of factors, i.e., the laser power, the travel speed of the laser beam, etc.

Immediately after the appearance of the method of laser controlled thermocracking (LCT) there followed attempts to use it for cutting some products with a complex curvilinear contour out of sheet glass [2]. However, during the transition of the laser beam path and the coolant from the straight line trajectory to a curvilinear one, a number of changes in the LCT conditions occur, which are to be taken into consideration to ensure the reliability and high quality of the process. To optimize the regimes of the laser controlled thermocracking by a curvilinear contour we have to consider the differences in the conditions of the surface material heating by the laser beam in the rectilinear and curvilinear sections of the trajectory with the use of a mathematical model of these processes [3].

\section{Calculations}

The task of the heating and subsequent cooling of the glass plates was solved numerically by the method of finite differences [4], and the nonlinearity of the surface cooling at the boiling water front was accounted for by adjusting the temperature at the previous step of the calculation. Let us write down the equation of thermal conductivity, the initial and boundary conditions for the characteristic regimes of LCT as follows:

$$
\left\{\begin{array}{l}
c \rho \frac{\partial T}{\partial t}-\lambda \Delta T=(1-R o) \frac{k P}{\pi A B} \cdot \exp \left(-\frac{2(x-v t)^{2}}{A^{2}}-\frac{2 y^{2}}{B^{2}}\right) \cdot \exp (-k z) \\
T(x, y, z, 0)=T o \\
\lambda \frac{\partial T}{\partial z}=\alpha(T)(T-T)
\end{array}\right.
$$

where $c, \rho, \lambda, R o, k, P, A, B, v$ are the heat capacity, density, thermal conductivity, reflection coefficient, absorption coefficient, the power of the laser radiation, the semi-axes of the ellipse spots of heating and movement speed, respectively. The heat transfer coefficient $\alpha(T)$ is determined experimentally [5]. For cutting around the circumference with a linear velocity $v=\omega R$ in the laser beam coordinates were introduced transformation $x \rightarrow$ $x \operatorname{Cos}(\omega t)+y \operatorname{Sin}(\omega t) ; y \rightarrow y \operatorname{Cos}(\omega t)-x \operatorname{Sin}(\omega t)$.

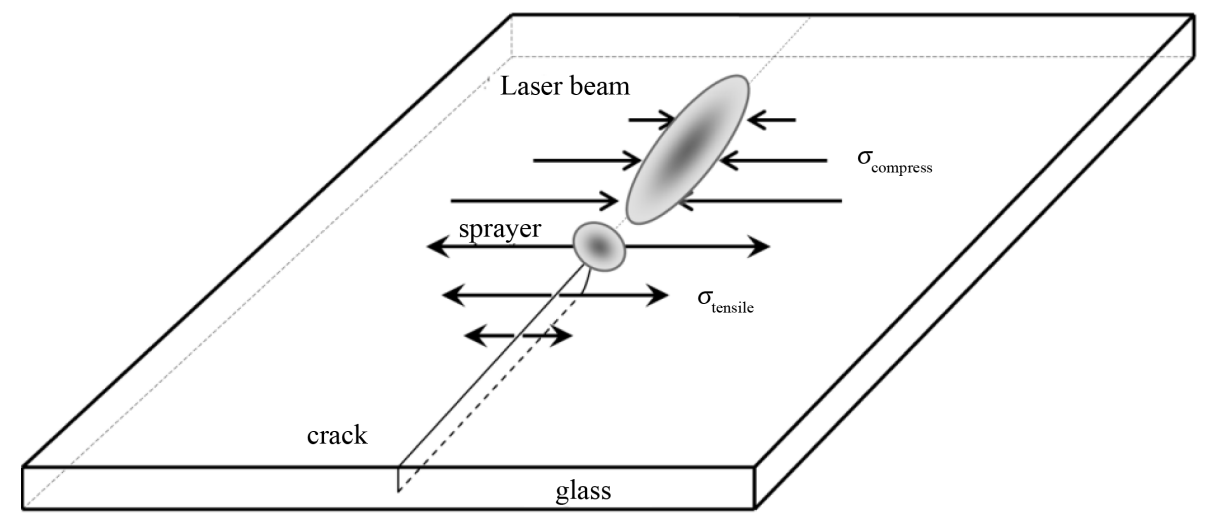

Figure 1. Physical model of the laser controlled thermocracking (LCT). 
We have performed calculations of temperature distribution on the surface of the glass at the straight section and curvilinear section of cutting with circle radius $R=16 \mathrm{~mm}$. We have considered two variants of calculating LCT parameters for the glass $1.8 \mathrm{~mm}$ thick in a straight cutting line and cutting by curvatures circumferentially with radius $R=16 \mathrm{~mm}$ with a long beam $2 A \times 2 B=12 \times 1.5 \mathrm{~mm}^{2}$ (Figure 2) and with a shorter elliptical beam $2 B \times 2 A=4 \times 2.5 \mathrm{~mm}^{2}$ (Figure 3). The calculations were carried out for the LCT parameters given in Table 1 .

In the transition from a linear section of cutting to curvilinear area with circle radius $R=16 \mathrm{~mm}$ it is necessary to change the parameters of the LCT. It is stipulated by the increase of the heating zone width, and, consequently, by a decrease in glass heating temperature. Due to the fact that the cutting of the product is carried out in a single technological cycle, changing the size of the laser beam and laser radiation power in the process of LCT is cumbersome and impractical. Therefore, to maintain the size of the laser beam and laser radiation power we must reduce the laser beam travel speed from 350 to $60 \mathrm{~mm} / \mathrm{sec}$ in the transition to the curved section; the speed drops six fold.

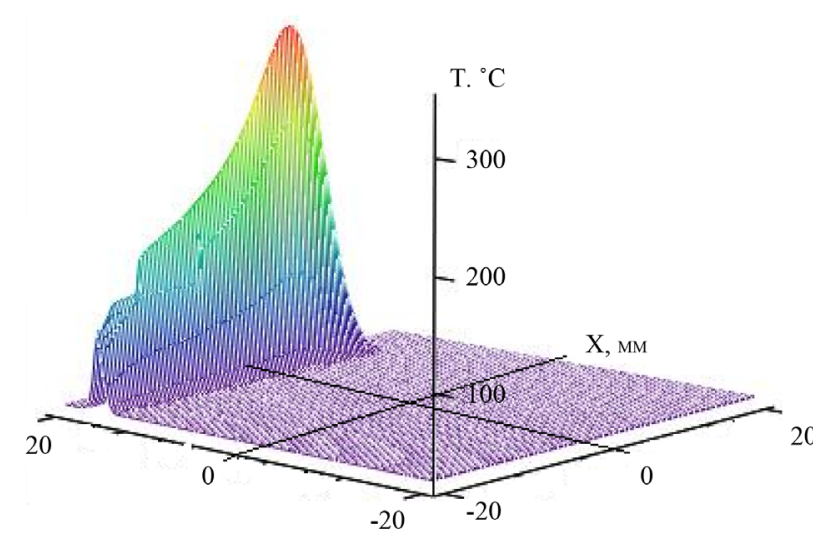

(a)

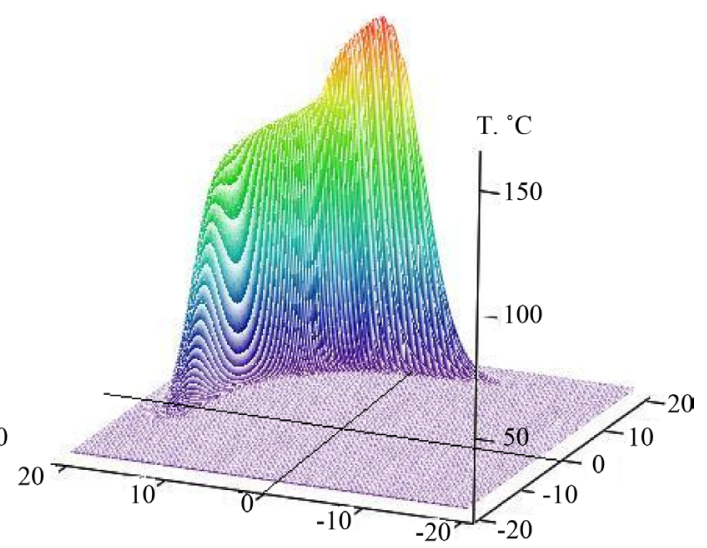

(b)

Figure 2. Temperature distribution on the surface of the glass at a straight section cutting (a) and in the area of cutting with a circle radius $R=16 \mathrm{~mm}$ (b) with a laser beam $2 A \times 2 B=12 \times 1.5 \mathrm{~mm}^{2}$.

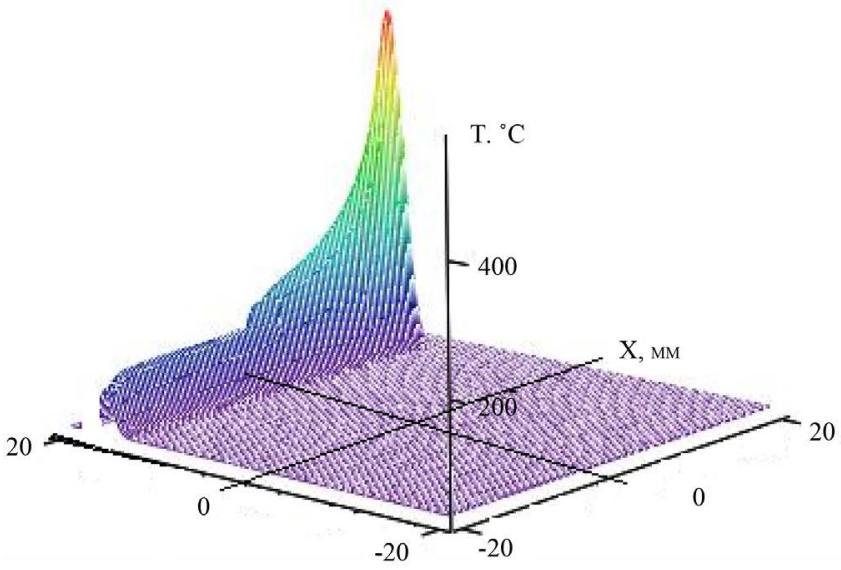

(a)

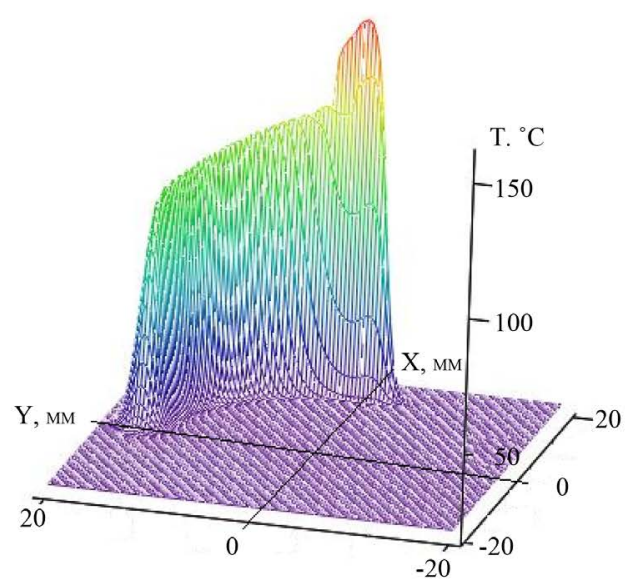

(b)

Figure 3. Temperature distribution on the glass surface in the straight section of cutting (a) and in the area of circumferential cutting with radius $R=16 \mathrm{~mm}$ (b) a laser beam $2 B \times 2 A=4 \times 2.5 \mathrm{~mm}^{2}$.

Table 1. The calculations were carried out for the LCT parameters.

\begin{tabular}{ccccc}
\hline \multicolumn{2}{c}{$2 A \times 2 B=12 \times 1.5 \mathrm{~mm}^{2}$} & & $2 A \times 2 B=4 \times 2.5 \mathrm{~mm}^{2}$ \\
\hline $\mathrm{P}, \mathrm{W}$ & $v$ straight, $\mathrm{mm} / \mathrm{s}$ & $v$ curvilinear, $\mathrm{mm} / \mathrm{s}$ & $\mathrm{P}, \mathrm{W}$ & $v$ straight, mm/s \\
\hline 100 & 350 & 60 & 80 & 150 \\
\hline
\end{tabular}


Now we consider cutting with the laser beam the size of $2 A \times 2 B=4 \times 2.5 \mathrm{~mm}^{2}$ with a short ellipse spot. We have calculated the temperature distribution on the surface of the glass (Figure 3 ) at a straight section of cutting (a) and a curved cutting section circumferentially with radius $R=16 \mathrm{~mm}$ (b).

When transiting to a curvilinear site and moving the glass in a circle of radius $R=16 \mathrm{~mm}$ it is necessary, just as in the previous case, to reduce the velocity of relative movement of the laser beam and the material. Keeping the size of the laser beam and the power of the laser radiation, it is necessary to reduce the travel speed from 150 to $50 \mathrm{~mm} / \mathrm{sec}$, i.e. 3 times.

On the one hand, the reduction in the length of the laser elliptical beam provides the reduction of the heating zone width in the curved sections of the cutting trajectory. On the other hand, the reduction in the length of the beam leads to a decrease in the rate of LCT. At the same time, with reduction of the curvilinear contour circumference radius the beam length influence on the LCT parameters increases.

Therefore, the most rational solution of the problems of the LCT products with a curvilinear contour is the use of special optics or scanning devices ensuring the form of the beam iterating the cut contour [6].

As can be seen from the diagram, the use of the laser beam, which repeats the form of the cutting contour, provides optimal LCT conditions. In the first place, we can maintain the optimal width of the heating zone in all parts of the trajectory of the relative movement of the laser beam and the material. The LCT speed in all parts remains constant. Such a way of cutting is very convenient when cutting discs of permanent sizes.

However, such a scheme of cutting has its weaknesses and limitations in the combined cutting, when we combine parts of the rectilinear cutting with areas of curvilinear cutting with a circle of a given radius. Therefore, the aim of this work is to optimize the parameters of laser controlled thermocracking (LCT) for cutting glass plates of any complex curvilinear contour.

\section{Cutting Glass Samples}

Glass cutting by a curvilinear contour is widely used in such industries as engineering (measuring devices, oculars, lenses, etc.), mobile devices (mobile phones, PDAs, laptops, tablets, displays, etc.), engineering industry (vehicles windows and mirrors, interior and wing mirrors).

An important advantage of glass usage as a structural material is its high performance characteristics, ecological cleanliness, optical transparency in the visible range of the radiation and the possibility of applying modern multi-touch technologies for touch devices. One such example is the tendency of introducing protective glass screens in Apple mobile devices.

The requirements to such products, the thickness of which, as a rule, amounts to $0.5-3 \mathrm{~mm}$, are very high, especially when they are used in optical and mobile devices. The key criteria are high strength, high accuracy of geometrical sizes (tolerance $\pm 0.02 \mathrm{~mm}$ ), as well as a smooth, defect-free, flawless and uniform edge. Currently, industries are widely employing traditional technology including a multi-level process of grinding and polishing the product edges by abrasive tools. The developed method of LCT allows not only obtaining products from glass which meet these requirements, but also the quality of which exceeds traditionally processed samples several times. The most important advantage is getting brittle products of high strength which is 5.5 times as high as the strength after the traditional processing.

This is ensured by the absence of stress concentrators and thermal hardening of the ice edge during the LCT. This means that the LCT process simultaneously provides the conditions for thermal hardening of the edge due to its heating to a temperature not exceeding the temperature of the glass softening (glass transition) $\mathrm{Tg}$, and the subsequent shock quenching of the heating zone by a coolant, here by an air-water jet. That is, modes of LCT automatically provide conditions for the air-to-liquid hardening of the glass edge.

To ensure high quality and high accuracy of geometrical sizes of the cut products it is necessary to observe the following conditions:

- high temporal stability of power and mode structure of laser radiation,

- high accuracy of positioning systems and moving the parts and products processed,

- spatial and temporal distribution of the coolant on the surface of the glass should be stable,

- optimal size of the laser beam and the power density of laser radiation,

- symmetric distribution of thermoelastic stresses at the line of cutting,

- optimal modes of laser thermal or mechanical follow-up cracking.

When cutting glass samples the coolant stain must be focused dead on the center relative to the line of separa- 
tion followed by a laser beam (Figure 4(b)). Should you fail to observe this condition, namely, when the coolant spots are left or right to the line of cutting (Figure 4(a) and Figure 4(b)), the symmetry of the thermoelastic stresses distribution gets broken, and consequently, the propagation of the crack is no longer perpendicular to the glass surface.

LCT technology in a curvilinear contour can be divided into two basic directions, viz. the cutting of a circle and alternating cutting both straightforwardly and with the specified radius of curvature (Figure 5).

The former (e.g. cutting discs) proves to be the simplest variant. To obtain a perpendicular crack it is enough to set the coolant stain circumferentially just once, strictly according to the line of the cut with the help of certain adjustments. Let us consider the process of alternating cutting in more detail (Figure 5).

The first condition for LCT by a curvilinear contour is the orientation of the elliptical beam at a tangent in each point of a curvilinear contour cutting. This is achieved by using a two-coordinate table to move along $\mathrm{x}$-axis and $\mathrm{y}$-axis and the rotary shaft $\mathrm{C}$ on which we fix optics and the nozzle. Such a scheme provides orientation of the laser beam at a tangent of the cut loop, but does not provide orientation of the coolant stain by the line of the cut to satisfy the condition as shown in Figure 4(b). When the optics turns at $90^{\circ}$ the coolant spot moves away from the cutting line (Figure 5(b)). Because thermoelastic stresses created in the cutting line are asymmetrical, the distribution of micro-cracks deep into the material will be non-perpendicular. This effect is noticeably manifested in the products with a curvature radius of $16 \mathrm{~mm}$ and less, and also on the spline lines.

To solve this problem, one should use two-axle rotary shaft; the optics will be mounted on one axis, and the nozzle on the other. Thus, the program is implemented to move coolant spots in the cutting process relative to the laser beam moving along the straight-line, covering the curvature radius, spline line or alternately changing curvilinear line (Figure 5(b)). These conditions will allow proper optimization of the thermoelastic stresses distribution along the cutting line, which will lead to the required result (Figure 4(c)).
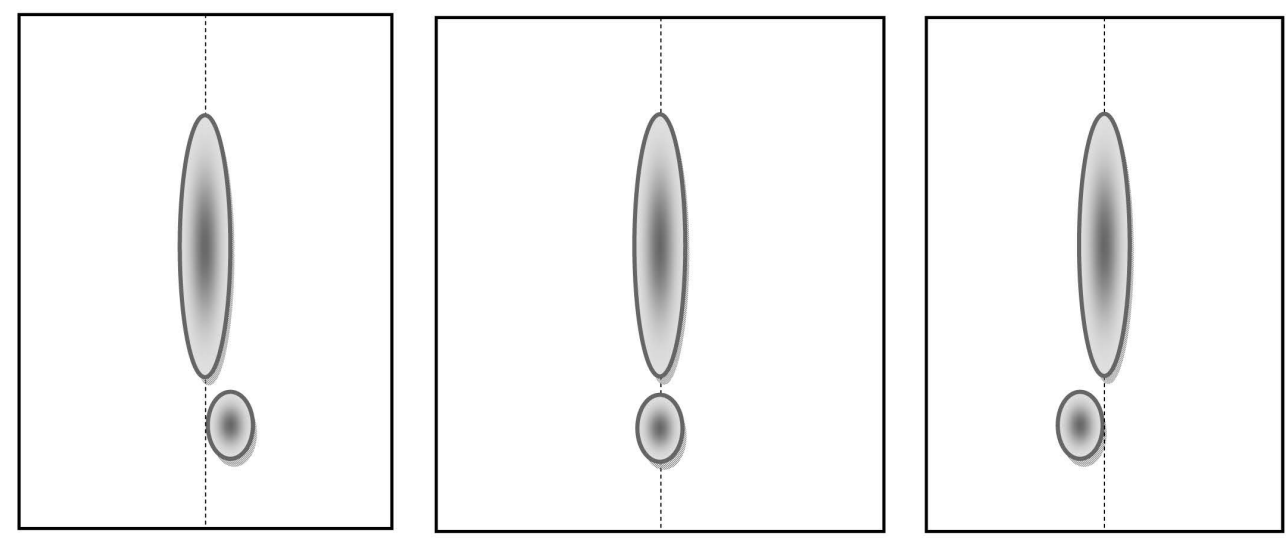

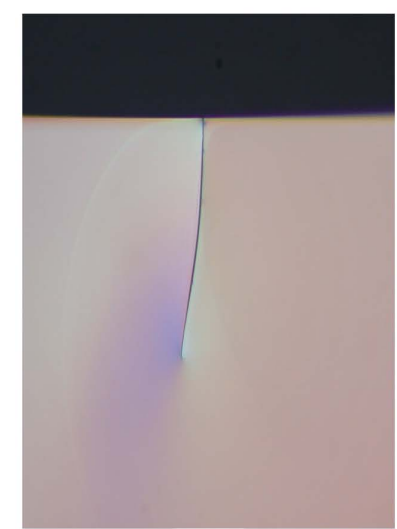

(a)

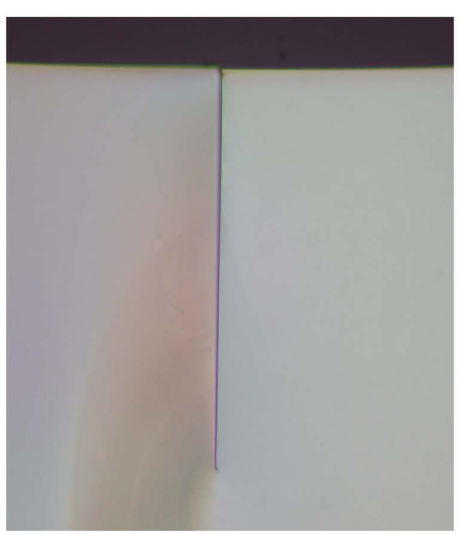

(b)

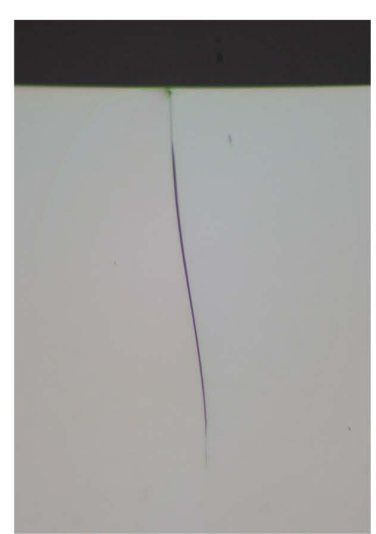

(c)

Figure 4. The distribution of cracks deep into the material depending on the location of the coolant relative to the laser beam. 


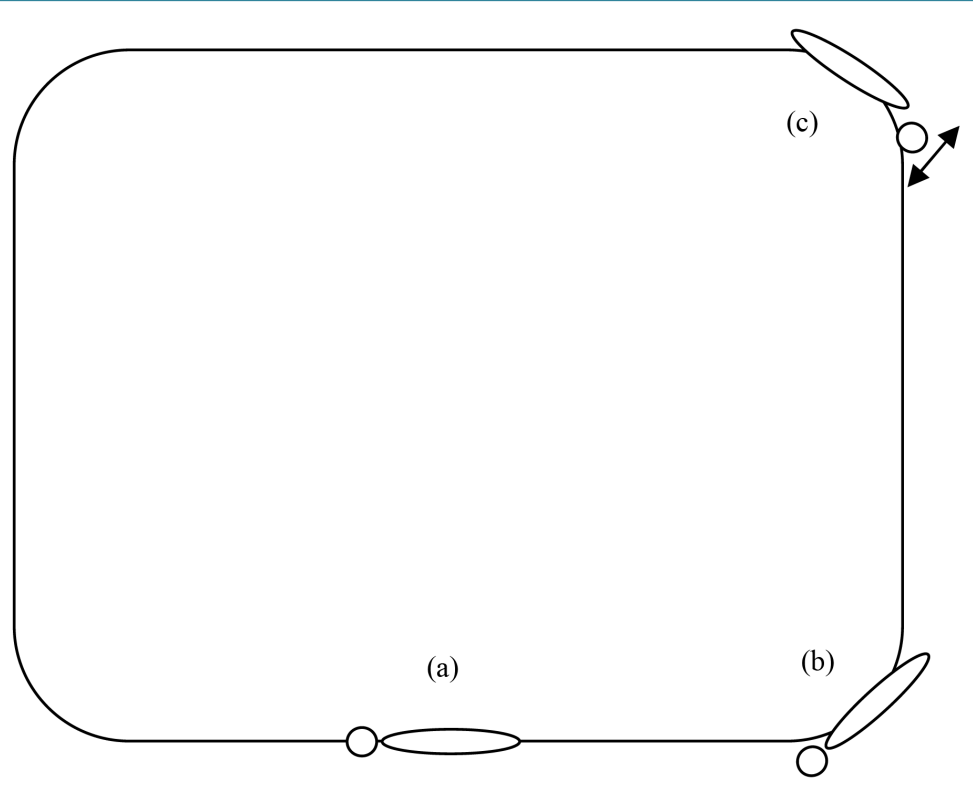

Figure 5. The scheme of LCT by a curvilinear contour of: (a) a straight line; (b) rounding; (c) curve with the possibility of displacing the coolant stains relative to the laser beam.

The consistency of the micro-crack depth along the whole contour is one of the conditions determining the accuracy of cutting the samples curvilinearly. This affects not only the so-called "cosmetic" indicators, but also the reproducibility of the process. The depth of cracks is known to be inversely proportional to the relative velocity of the laser beam motion [3]. Thus, to ensure consistency of the micro-cracks depth in the range of \pm 10 micrometers along the whole contour we have to optimize the linear velocity at each point of the curvilinear contour. It is a necessary procedure owing to the fact that when you rotate the laser beam the ellipses axes do not coincide with the cutting line, the distribution of thermoelastic stresses goes irregular, and the heating is insufficient to create a gradient of temperatures.

To obtain a high-quality, perpendicular through-crack one should re-heat the cutting line with the help of laser radiation. This requires compliance with the symmetry of the thermoelastic stresses distribution along the cutting line. Figure 6 illustrates the character of the through-cracks development in the glass $1.1 \mathrm{~mm}$ thick, depending on the location of the laser beam relative to the micro-cracks when re-heating the cutting line. As you can see in the photos in Figures 6(a)-(c), if a micro-crack is perpendicular the high-quality follow-up cracking occurs only when the laser beam is strictly symmetrically oriented to the line of the cut (Figure 6(b)). During the re-heating even a slight shift of the laser beam to the left or right from the cutting line can get the additional cracking line distorted (Figure 6(a) and Figure 6(b)).

Thus, taking into account all the above-mentioned parameters of the LCT process and their interactions which determine the accuracy and quality of cutting we managed to optimize the process of laser controlled thermocracking of glass by a curvilinear contour.

This program, which determines the position of the laser beam and the coolant to obtain products of the required size, takes into account the curvature radius, the beam length, and the distance from the center of the coolant stain to the center of the beam. Knowing these parameters, you can quickly configure the process to get the expected results.

Basing on the patent license, Foxconn Technology Group (Taiwan) has developed a laser industrial unit for cutting glass by curvilinear contour and beveling the edges in a single technological cycle (Figure 7).

Figure 8 features photos of glass products for iPhone (a), iPod (b) and notebooks (c) cut by a curvilinear contours employing LCT method with a chamfered edge.

\section{Conclusion}

The performed research has enabled optimizing the modes of the laser controlled thermocracking by a curvilinear 

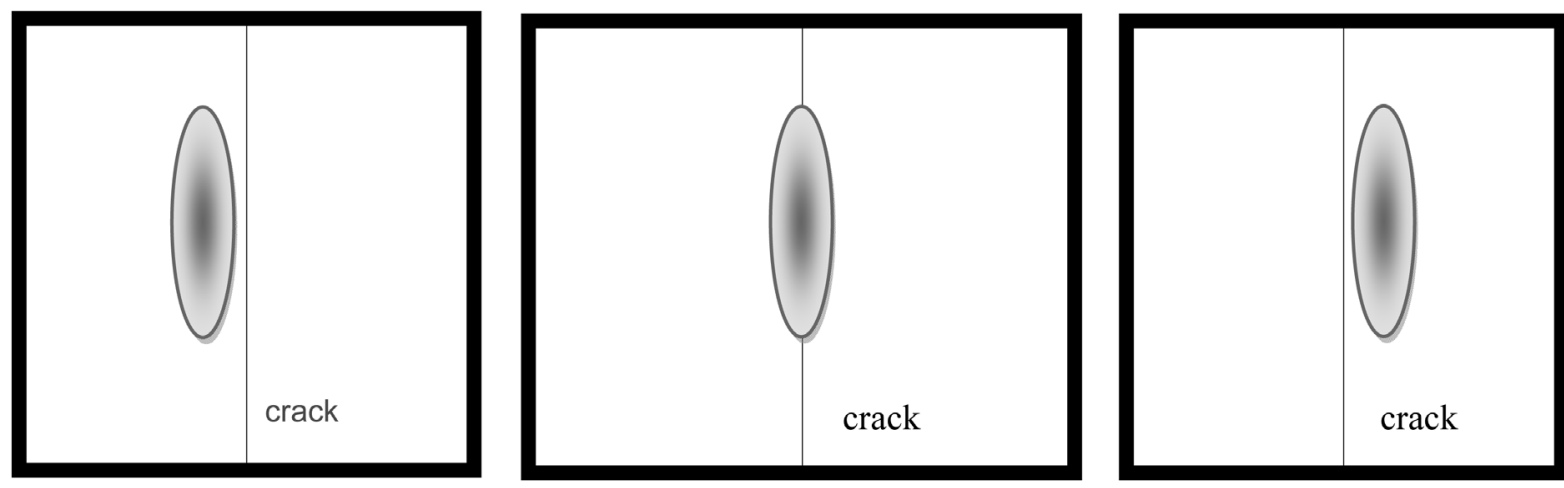

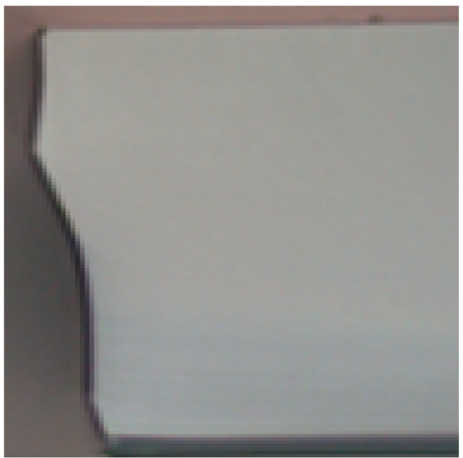

(a)

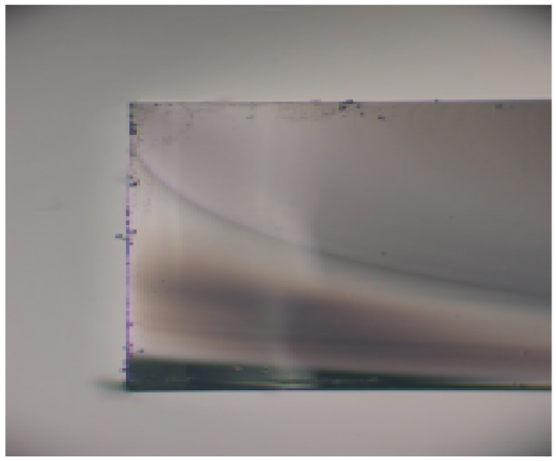

(b)

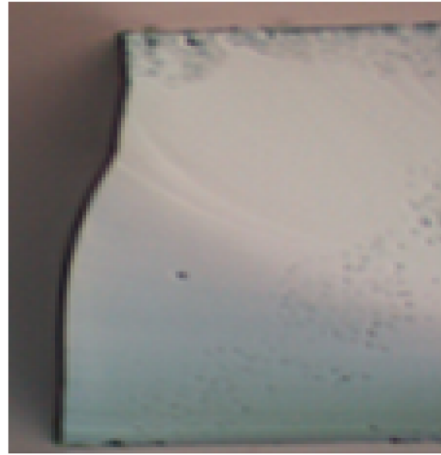

(c)

Figure 6. The distribution of through cracks in the glass $1.1 \mathrm{~mm}$ thick depending on the location of the laser beam relative to the cracks.

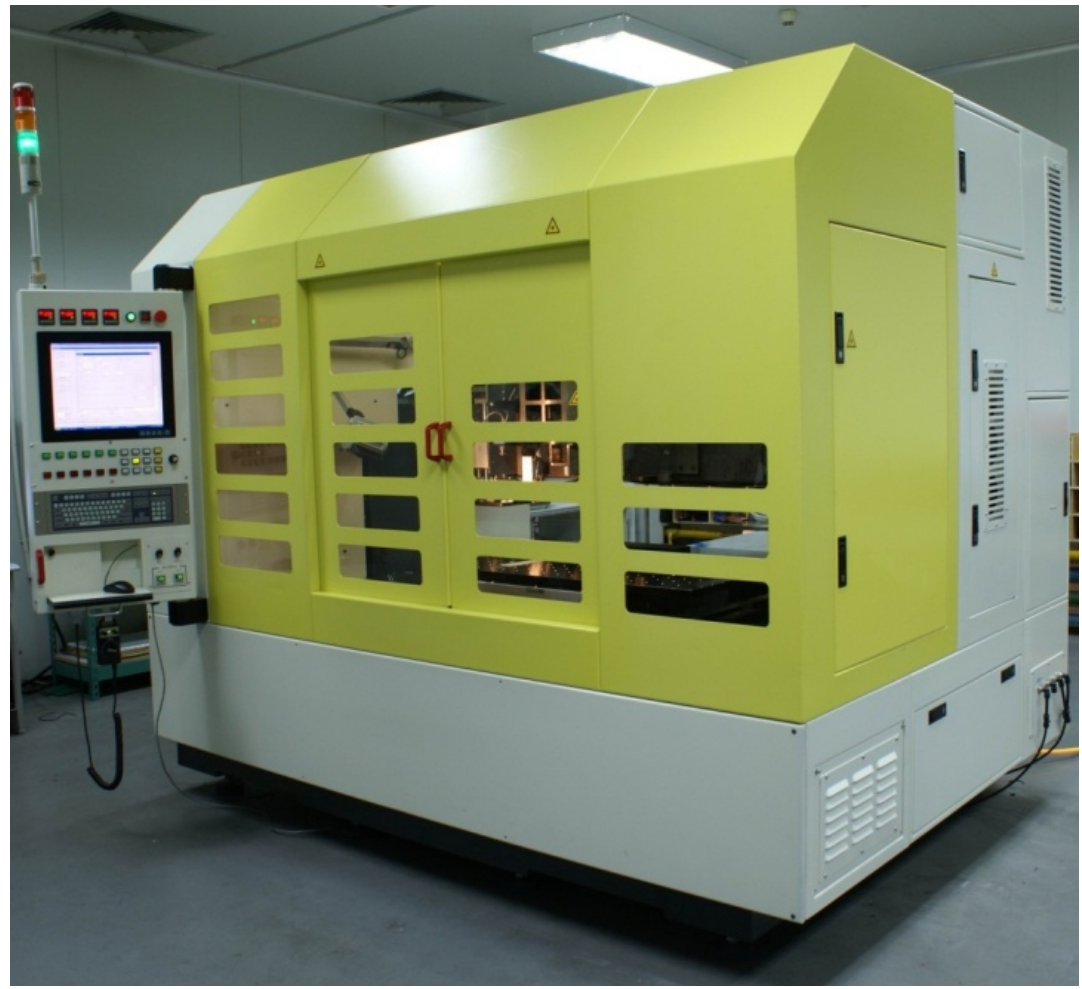

Figure 7. A laser industrial unit of Foxconn technology group to cut glass by a curvilinear contour. 


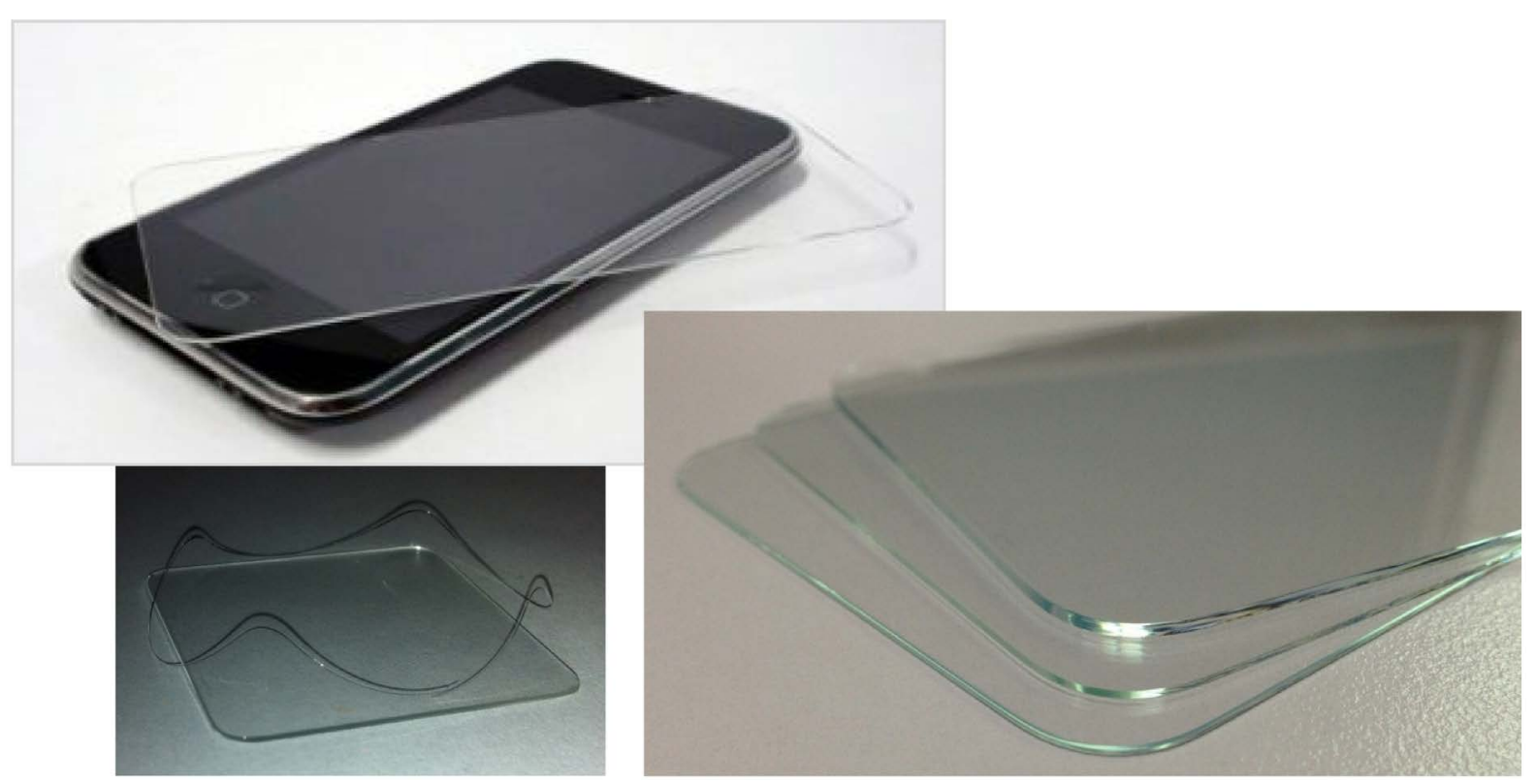

Figure 8. Photos of protective glasses for the iPhone and iPod, the cut of Corning Gorilla glass using LCT method.

contour and implementing the LCT process in production in Russia and abroad. Foxconn Technology Group (Taiwan) has developed and launched industrial laser technological units in production.

\section{References}

[1] Kondratenko, V.S., Gindin, P.D., Trubienko, O.V., Muchi, H. and Naumov, A.S. (2009) Laser Strengthening of the Edge of Glass. Journal of Optical Technology, 76, 733-736. http://dx.doi.org/10.1364/JOT.76.000733

[2] Kondratenko, V.S., Tanaseyichuk, A.S. and Shershnev, Ye.V. (1986) The Peculiarities of the Process of Laser Controlled Thermocracking of Float Glass by a Curvilinear Contour. Electronic Technique. Series 11, 4, 46-49.

[3] Kondratenko, V.S., Borisovsky, V.E., Gindin, P.D. and Naumov, A.S. (2008) New Technologies of Laser Machining of Parts for Optical Engineering. Instruments, 3, 36-39.

[4] Korn, G. and Korn, T. (1977) Handbook of Mathematics. M., Nauka.

[5] Isachenko, V.P., Osipova, V.A. and Sukomel, A.S. (1968) Heat Transfer. M., Energoizdat.

[6] Kondratenko, V.S. (1996) Method of Cutting Non-Metallic Materials and a Device for Carrying It Out. Patent No. WO9620062. 